\section{Nomenclature of the tendon hierarchy: An overview of inconsistent terminology and a proposed size-based naming scheme with terminology for multi-muscle tendons}

In recent decades, tendon biomechanics research has increased markedly and is beginning to reveal how complex structural variations through the tendon hierarchy contribute to varied tissue mechanical properties and meet different functional needs. However, as research in this area increases, inconsistencies in terminology describing the tendon hierarchy are becoming increasingly apparent. We suggest that adoption of consistent nomenclature for describing tendon hierarchies will promote greater communication and collaboration between researchers. At the very least, the increased understanding of tendon morphology dictates an open discussion of language inconsistencies. The purposes of this letter are to first: draw attention to the variations in nomenclature currently used, second: propose an adapted size-based naming system for the tendon hierarchy, and third: propose a term to describe the specialized substructure present in multi-muscle tendons such as the Achilles.

The collagen hierarchy of tendon has long been established, and was categorized by Kastelic et al. (1978) into six levels: 'tropocollagen', 'microfibril', 'subfibril', 'fibril', 'fascicle' and 'tendon'. Since this early publication, however, such terminology has only been partially adopted, with subsequent authors introducing their own, and at times contradictory, naming conventions. For instance, Kannus (2000) uses the term 'fascicle' to describe the substructure two levels below the whole tendon characterized by observable longitudinal banding. In contrast, Kastelic et al. (1978) use the term 'fibril' for the level with longitudinal banding and 'fascicle' for the first substructure below the tendon (Kannus, 2000) (Fig. 1). Additionally, various groups have reported different numbers of hierarchical levels, for example, O'Brien (1997) reported five and Wang (2006) reported seven (Fig. 1). This disagreement may relate to methodological variations; probing the tendon hierarchy is challenging, owing to the large aspect ratios of constituent components and limited sensitivity of different technical approaches to clearly identify all levels. However, it is also possible some of these differences reflect real structural variations in different tendons (Kannus, 2000). For example, recent evidence suggests that even within the same species, tendons with different primary functions exhibit specific structural differences (Thorpe et al., 2012), making it likely that there are also differences across species, particularly between those of different sizes, where smaller animals with smaller tendons may have fewer hierarchical levels than large animals.

Given the possibility that the tendon hierarchy differs across tendons and animals, we posit that the most sensible way to objectively identify the hierarchical levels below the whole tendon is based on structure diameter, with terminology preference given to the seminal and oft-cited work of Kastelic at al. (1978). The debate concerning collagen molecular packing is beyond the scope of this short article, but several hierarchical levels and their diameters can be identified across tendons in general. The collagen molecule (tropocollagen), for instance, is around $1.5 \mathrm{~nm}$ in diameter and aggregates in groups of five to make microfibrils (Orgel et al., 2006), which in turn aggregate to form collagen fibrils. Collagen fibrils are often considered the fundamental building block across different connective tissues and may be seen with electron microscopy (Parry et al., 1978), with diameters ranging from 50-500 nm. Fascicles are most often described as the clearly defined units visible to the eye, where a diameter range of 50$400 \mu \mathrm{m}$ captures well the variation across animals (Screen, 2009). Like these three examples, using naming standards based on measured diameter provides objectivity to the nomenclature and grounds the use of language in a common measurement.

The remainder of this letter is focused on an additional macroscale hierarchical level observed in tendons that are shared between multiple muscles. One notable example of this arrangement is the Achilles tendon, which is actuated by three distinct muscle bellies (medial gastrocnemius, lateral gastrocnemius, and soleus) fused distally into a single tendon (Bojsen-Møller and Magnusson, 2015; Edama et al., 2014). Although the portions of the whole tendon arising from each muscle are tightly fused, they are morphologically distinguishable and can be dissected (Sarrafian, 1993; Szaro et al., 2009). Further, experimental evidence suggests that this structural arrangement enables the tendon to maintain some combination of independent and cohesive motion (Bojsen-Møller et al., 2004; Slane and Thelen, 2014). We propose that the Achilles, and some other 'shared' tendons, represent a special class of tendons defined by this additional macro-scale hierarchical level. Previous authors have used the term 'fascicle' to describe this level (Edama et al., 2014; Szaro et al., 2009) (Fig. 1 (B)), but the separate portions of these tendons are composed of many fascicles arising from the actuating muscles. 'Fascicle bundle' (Bojsen-Møller and Magnusson, 2015) (Fig. 1(B)) may thus be considered an appropriate term, but the authors have preliminary unpublished evidence suggesting that fascicles form bundles even in tendons with only one muscle attachment (e.g. equine superficial digital flexor tendon), hence the term might be more appropriately reserved for these hierarchical units (see Fig. 1(C)). We propose the term 'subtendon' to refer to distinct portions of multi-muscle tendons. This term is straightforward and indicates a structure which is part of the whole tendon, but emanates from and transmits forces from a distinct muscle belly (e.g. soleus subtendon). While the authors' motivation for the term 'subtendon' is in describing Achilles tendon substructures, other tendons with a structural arrangement similar to the Achilles (e.g. quadriceps tendon) may benefit from use of the term 'subtendon' to distinguish portions of the tendon corresponding to different 
A. Literature nomenclature for tendon hierarchy

Kastelic et al. (1978)

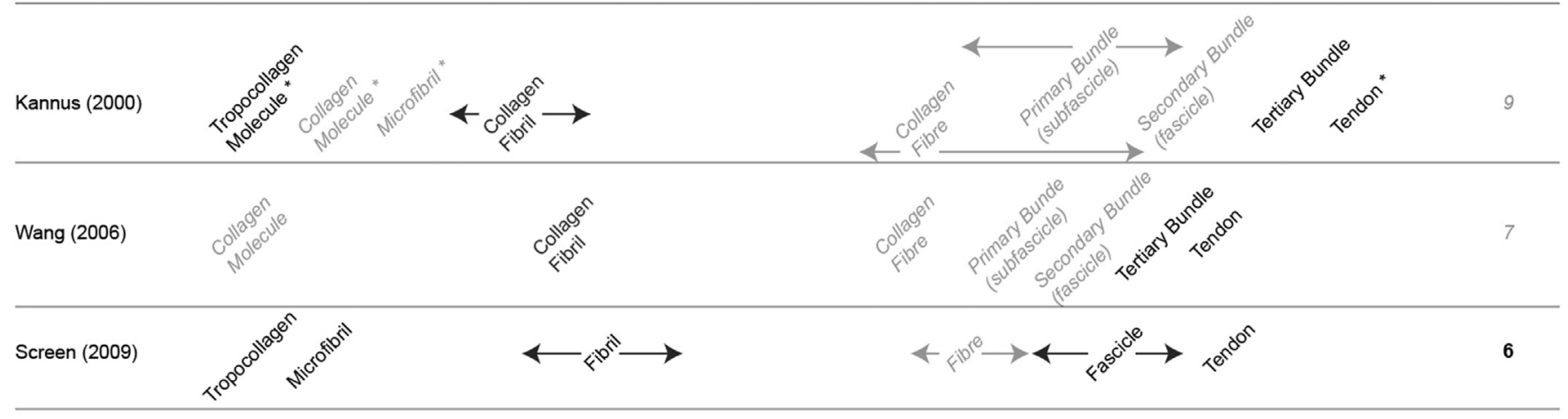

\section{B. Nomenclature for human Achilles sub-structure}

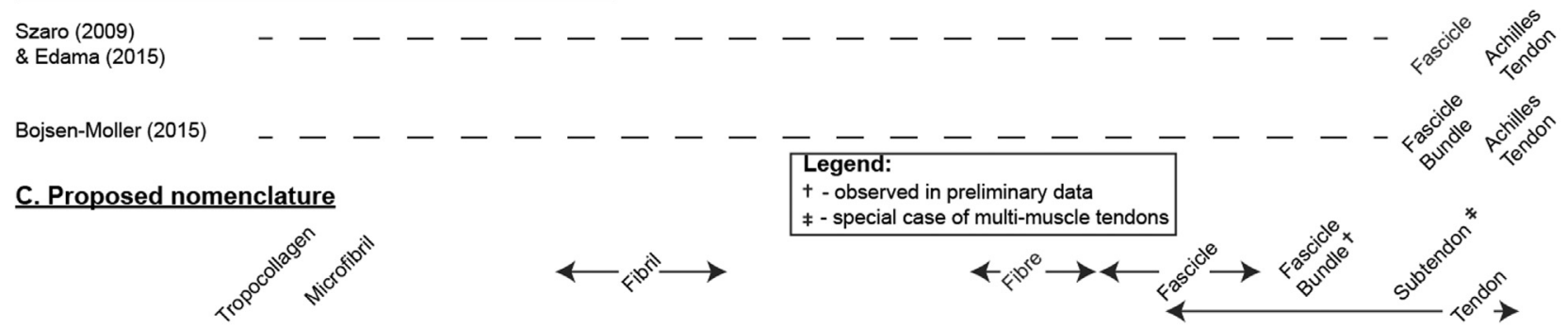

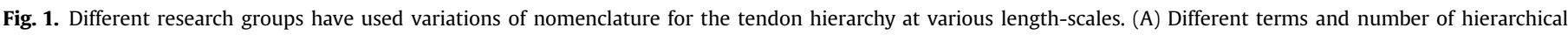

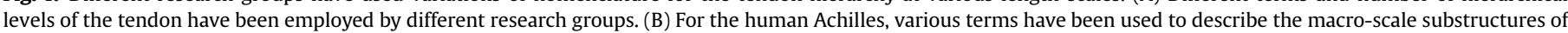

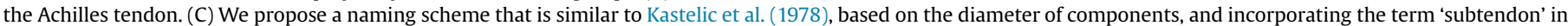

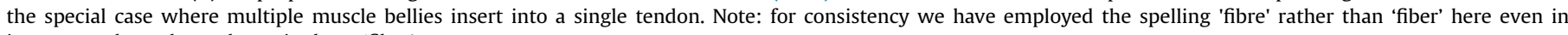
instances where the authors cited use 'fiber'.

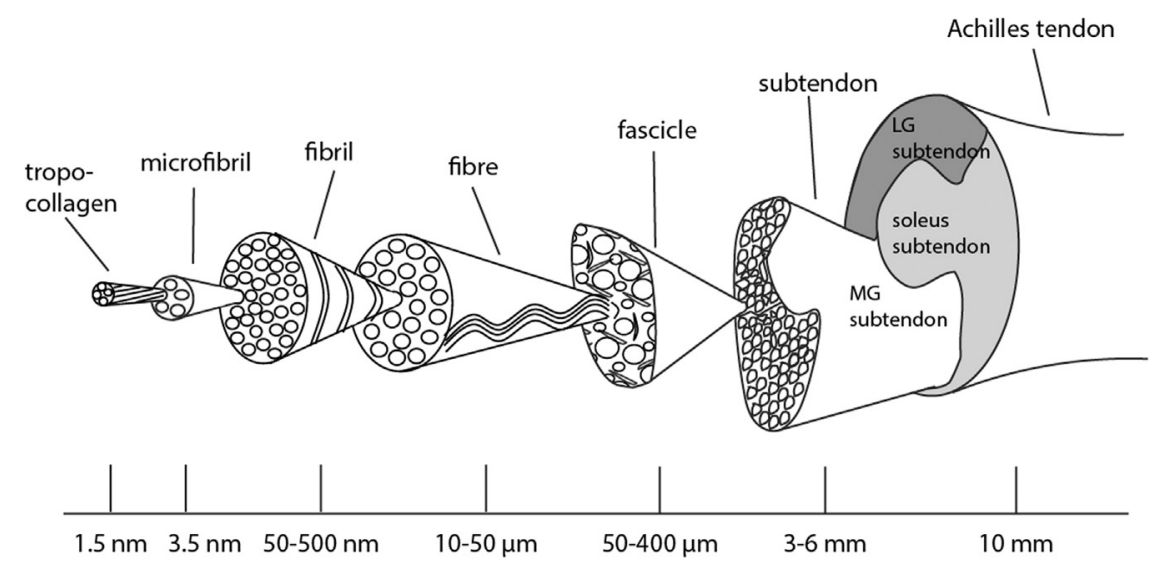

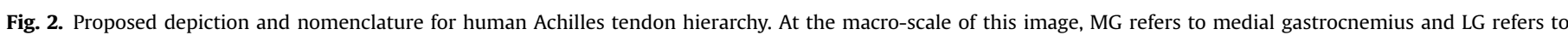
lateral gastrocnemius, these being the muscle bellies corresponding to the labeled subtendons.

muscle bellies. Further discussion is warranted on whether terminology for all multi-muscle tendons should be united.

In conclusion, we feel that as tendon research advances, it is increasingly important for researchers to use common terminology that is defined precisely. A naming scheme based on structural diameter offers objectivity in defining hierarchical levels, and we propose that a size-based naming scheme with terminology rooted in the seminal work of Kastelic et al. (1978) will serve this purpose (Fig. 2). At the macro-scale, we introduce the term 'subtendon' to refer to the hierarchical level observed in the Achilles 
and other tendons that are actuated by more than one muscle belly. This term distinguishes distinct components of multi-muscle tendons and clearly specifies the anatomical arrangement of these components and the muscles from which they emanate. We hope that this letter may encourage researchers to aim for the use of unified language to drive forward this field. As always, we encourage dialog from other researchers on this relevant issue.

\section{Conflicts of interest}

We have no conflicts of interest to declare.

\section{Acknowledgments}

We gratefully acknowledge fellowship support provided by the Whitaker International Program.

\section{References}

Bojsen-Møller, J., Hansen, P., Aagaard, P., Svantesson, U., Kjaer, M., Magnusson, S.P., 2004. Differential displacement of the human soleus and medial gastrocnemius aponeuroses during isometric plantar flexor contractions in vivo. J. Appl. Physiol. 97, 1908-1914. http://dx.doi.org/10.1152/japplphysiol.00084.2004.
Bojsen-Møller, J., Magnusson, S.P., 2015. Heterogeneous loading of the human Achilles tendon in vivo. Exerc. Sport Sci. Rev. 43, 190-197. http://dx.doi.org/ 10.1249/JES.0000000000000062.

Edama, M., Kubo, M., Onishi, H., Takabayashi, T., Inai, T., Yokoyama, E., Hiroshi, W., Satoshi, N., Kageyama, I., 2014. The twisted structure of the human Achilles tendon. Scand. J. Med. Sci. Sports, 1-7. http://dx.doi.org/10.1111/sms.12342.

Kannus, P., 2000. Structure of the tendon connective tissue. Scand. J. Med. Sci. Sports 10, 312-320. http://dx.doi.org/10.1034/j.1600-0838.2000.010006312.x.

Kastelic, J., Galeski, A., Baer, E., 1978. The multicomposite structure of tendon Connect. Tissue Res. 6, 11-23. http://dx.doi.org/10.3109/03008207809152283.

O'Brien, M., 1997. Structure and metabolism of tendons. Scand. J. Med. Sci. Sports 7, 55-61. http://dx.doi.org/10.1111/j.1600-0838.1997.tb00119.x.

Orgel, J.P.R.O., Irving, T.C., Miller, A., Wess, T.J., 2006. Microfibrillar structure of type I collagen in situ. Proc. Natl. Acad. Sci. USA 103, 9001. http://dx.doi.org/10.1073/ pnas.0502718103.

Parry, D.A., Barnes, G.R., Craig, A.S., 1978. A comparison of the size distribution of collagen fibrils in connective tissues as a function of age and a possible relation between fibril size distribution and mechanical properties. Proc. R. Soc. Lond. Ser. B Contain. Pap. Biol. Charact. R. Soc. 203, 305-321.

Sarrafian, S.K., 1993. Calcaneal (achilles) tendon, In: Anatomy of the Foot and Ankle: Descriptive Topographic Functional, J.B. Lippincott Company, Philadelphia, pp. 280-282.

Screen, H.R., 2009. Hierarchical approaches to understanding tendon mechanics. J. Biomech. Sci. Eng. 4, 481-499. http://dx.doi.org/10.1299/jbse.4.481.

Slane, L.C., Thelen, D.G., 2014. Non-uniform displacements within the Achilles tendon observed during passive and eccentric loading. J. Biomech. 47, 2831-2835. http://dx.doi.org/10.1016/j.jbiomech.2014.07.032.

Szaro, P., Witkowski, G., Smigielski, R., Krajewski, P., Ciszek, B., 2009. Fascicles of the adult human Achilles tendon - an anatomical study. Ann. Anat. 191, 586-593. http://dx.doi.org/10.1016/j.aanat.2009.07.006 10.1016/j.aanat.2009.07.006.

Thorpe, C.T., Udeze, C.P., Birch, H.L., Clegg, P.D., Screen, H.R., 2012. Specialization of tendon mechanical properties results from interfascicular differences. J. R. Soc. Interface 9, 3108-3117.

Wang, J.H.-C., 2006. Mechanobiology of tendon. J. Biomech. 39, 1563-1582. http: //dx.doi.org/10.1016/j.jbiomech.2005.05.011. 